\title{
hCLOCK induction by hypoxia promotes inflammatory responses by activating the $\mathrm{NF}-\kappa \mathrm{B}$ pathway
}

\author{
XIAO TANG $^{1 *}$, DAQIAO GUO ${ }^{*}$, CHANGPO LIN $^{1}$, ZHENYU SHI $^{1}$, RUIZHE QIAN $^{2}$, \\ WEIGUO FU ${ }^{1}$, JIANJUN LIU ${ }^{3}, \mathrm{XU} \mathrm{LI}^{3}$ and LONGHUA FAN ${ }^{3}$ \\ ${ }^{1}$ Department of Vascular Surgery, Institute of Vascular Surgery, Zhongshan Hospital, Fudan University; \\ ${ }^{2}$ Department of Physiology and Pathophysiology, Fudan University Shanghai Medical College; ${ }^{3}$ Department of \\ Vascular Surgery, Qingpu Branch of Zhongshan Hospital, Fudan University, Shanghai 200032, P.R. China
}

Received November 25, 2015; Accepted November 25, 2016

DOI: $10.3892 / \mathrm{mmr} .2017 .6127$

\begin{abstract}
The expression and secretion of inflammation-associated cytokines are induced by hypoxia. Circadian locomotor output cycles protein kaput (CLOCK) has previously been shown to activate the nuclear factor- $\kappa \mathrm{B}$ $(\mathrm{NF}-\kappa \mathrm{B})$ pathway, which is a key transcription factor during hypoxia. The present study evaluated the role of the NF- $\kappa \mathrm{B}$ pathway in the CLOCK-induced inflammatory response. Under hypoxic conditions, the expression levels of NF- $\mathrm{NB}$ and proinflammatory cytokines, including interleukin (IL)-1, IL-1 $\beta$, IL-6, intercellular adhesion molecule 1, cyclooxygenase 2 and tumor necrosis factor alpha, were significantly increased compared with under control conditions. Conversely, human umbilical vein endothelial cells (HUVECs) that were transfected with small hairpin RNA against human CLOCK exhibited reversed effects. Furthermore, inhibition of $\mathrm{NF}-\kappa \mathrm{B}$ with pyrrolidine dithiocarbamate (PDTC) reduced the expression of proinflammatory cytokines in HUVECs treated under hypoxic conditions. In addition, the CLOCK-induced inflammatory response was abolished with PDTC treatment. These findings suggest that the mechanism by which CLOCK induces inflammation mainly involves activation of the $\mathrm{NF}-\kappa \mathrm{B}$ signaling pathway.
\end{abstract}

\section{Introduction}

Circadian rhythms are endogenous, cyclical 24-h variations that are associated with numerous physiological processes. These rhythms are generated by circadian clocks, which are located in most cell types, including cells of the cardiovascular

Correspondence to: Professor Longhua Fan, Department of Vascular Surgery, Qingpu Branch of Zhongshan Hospital, Fudan University, 1158 Park Road, Qingpu, Shanghai 200032, P.R. China

E-mail: fanlonghuazs@163.com

${ }^{*}$ Contributed equally

Key words: circadian locomotor output cycles protein kaput, hypoxia, inflammatory response, nuclear factor- $\kappa \mathrm{B}$ system $(1,2)$. The mammalian circadian clock consists of a network of transcriptional and translational feedback loops (3). Two basic helix-loop-helix-PAS domain transcription factors, circadian locomotor output cycle protein kaput (CLOCK) and brain and muscle Arnt-like protein 1, are at the core of the major circadian loop. Clock-controlled genes are able to encode various proteins, and they markedly influence cellular functions.

Aberrations in the circadian clock system have pathological consequences. Disruptions to the circadian rhythm and defects in circadian clock genes are known to be associated with certain human malignancies $(4,5)$. Previous studies have indicated that biological oscillations driven by the circadian clock are able to influence endothelial dysfunction, pathological vascular remodeling and thrombosis, which may ultimately result in vascular disease $(6,7)$. Our previous study revealed that the upregulation of CLOCK in the vessel walls of veins may be involved in the pathogenesis and progression of venous disease (8). However, the exact molecular mechanism underlying this circadian control and the induction of inflammation remain largely unknown.

Nuclear factor- $\kappa \mathrm{B}(\mathrm{NF}-\kappa \mathrm{B})$ belongs to a family of constitutive and inducible transcription factors that act through diverse target genes. The NF- $\mathrm{B}$ pathway serves an important role in controlling the response to cellular stress, and it is also involved in cell division, transformation, survival, apoptosis, inflammation and immunity (9-11). Transcription factor p65 is a member of the NF- $\mathrm{NB}$ family, which has an important function in inflammation and the immune response (12). Research over the past decade has identified novel molecular links between the circadian clock and the NF- $\mathrm{B}$ pathway $(13,14)$. Our previous study demonstrated that CLOCK may activate the $\mathrm{NF}-\kappa \mathrm{B}$ pathway (14); however, in spite of the substantial evidence supporting the existence of crosstalk between the circadian clock and the $\mathrm{NF}-\kappa \mathrm{B}$ pathway, further investigation is required to identify the associations and mechanisms. The present study aimed to evaluate the crucial role of the $N F-\kappa B$ pathway in the CLOCK-induced inflammatory response at the molecular level.

\section{Materials and methods}

Cell culture and treatment. Human umbilical vein endothelial cells (HUVECs) were purchased from the American Type 
Culture Collection (Manassas, VA, USA) and maintained using an Endothelial Cell Growth Medium-2 BulletKit (Lonza Group AG, Basel, Switzerland) containing 10\% fetal bovine serum (Gibco; Thermo Fisher Scientific, Inc., Waltham, MA, USA), $100 \mathrm{U} / \mathrm{ml}$ penicillin and $100 \mu \mathrm{g} / \mathrm{ml}$ streptomycin at $37^{\circ} \mathrm{C}$ in an atmosphere containing $5 \% \mathrm{CO}_{2}$ and $95 \%$ room air. The Xvivo Closed Incubation system (Xvivo system 300C; BioSpherix, Parish, NY, USA) was used to accurately maintain different oxygen tensions in different chambers. Pyrrolidine dithiocarbamate (PDTC), an inhibitor of NF- $\mathrm{KB}$, was purchased from Sigma-Aldrich (Merck Millipore, Darmstadt, Germany) and a stock solution $(200 \mathrm{mmol})$ was prepared using distilled water. After $24 \mathrm{~h}$ of culture, the cells were divided into separate chambers with $5 \% \mathrm{O}_{2}$ and further incubated for 12 or $24 \mathrm{~h}$; subsequently, they were harvested for measurement.

Preparation of the retroviral vector. Gain- and loss-of-function of human CLOCK (hCLOCK) was established as previously described (15). The hCLOCK-coding sequence was amplified and subcloned into the pGV186 retroviral vector (GeneChem Co., Ltd., Shanghai, China). The short hairpin RNA (shRNA) targeting hCLOCK was designed and constructed by Shanghai GeneChem Co., Ltd. (Shanghai, China). Vector and shRNA sequences, as well as successful insertions were confirmed by DNA sequencing (Biosune Biotechnology Co., Ltd., Shanghai, China). Scramble control (SCR) served as a control for hCLOCK knockdown. For the production of viral particles, $293 \mathrm{~T}$ cells were co-transfected with the lentiviral vectors, psPAX2 and pMD2.G (Addgene, Inc., Cambridge, MA, USA) using X-tremeGENE Transfection Reagent (Roche Diagnostics, Basel, Switzerland), according to the manufacturer's protocol. The viral supernatant was collected $48 \mathrm{~h}$ post-transfection and passed through a $0.45-\mu \mathrm{m}$ filter (Sartorius AG, Göttingen, German). HUVECs were infected with lentiviral particles containing $8 \mu \mathrm{g} / \mathrm{ml}$ Polybrene Transfection Reagent (Merck Millipore, Darmstadt, Germany). Stable overexpression or knockdown of hCLOCK was confirmed by western blot analysis. After $24 \mathrm{~h}$ of cultivation in conventional cell culture, the cells were divided into separate chambers containing $5 \%$ $\mathrm{O}_{2}$ and incubated for 12 or $24 \mathrm{~h}$ prior to harvesting.

Enzyme-linked immunosorbent assay (ELISA). The expression levels of proinflammatory cytokines, including interleukin (IL)-1 $\beta$ (catalog no. DLB50), IL-6 (catalog no. D6050) and tumor necrosis factor- $\alpha$ (TNF- $\alpha$; catalog no. DTA00C), in the supernatants were measured using commercial ELISA kits (R\&D Systems Inc., Minneapolis, MN USA) according to the manufacturer's protocol.

Western blot analysis. The relative expression levels of hCLOCK, IL-1 $\beta$, IL-6, intercellular adhesion molecule 1 (ICAM-1), cyclooxygenase 2 (COX-2), TNF- $\alpha$, phosphorylated-p65 (p-p65) and $\beta$-actin were measured by western blot analysis using standard methods, as previously described (15). Briefly, total cell proteins were extracted using Radioimmunoprecipitation Assay Reagent (Cell Signaling Technology, Inc., Danvers, MA, USA), and concentrations were measured using the Bicinchoninic Acid Protein Assay kit (Bio-Rad Laboratories, Inc., Hercules, CA, USA). Equal amounts of protein $(50 \mu \mathrm{g})$ were separated by $10 \%$ sodium dodecyl sulfate-polyacrylamide gel electrophoresis and transferred to polyvinylidene difluoride membranes (Bio-Rad Laboratories, Inc.). Membranes were blocked with 5\% skimmed milk in TBS containing $0.1 \%$ Tween-20 at room temperature for $1 \mathrm{~h}$. A mouse monoclonal anti-hCLOCK (1:500; catalog no. ab98948) antibody was purchased from Abcam (Cambridge, MA, USA). Rabbit polyclonal anti-IL-6 antibody (1:1,000; catalog no. 21865-1-AP) was purchased from Proteintech Group, Inc. (Chicago, IL, USA). Rabbit polyclonal anti-COX2 (1:1,000; catalog no. 4842), rabbit polyclonal anti-IL-1 $\beta(1: 1,000$; catalog no. 12703), rabbit polyclonal anti-ICAM-1 (1:1,000; catalog no. 4915), rabbit monoclonal anti-TNF- $\alpha(1: 1,000$; catalog no. 6945) and rabbit monoclonal anti-p-NF-кB p65 (Ser536; 1:1,000; clone, 93H1, catalog no. 3033) antibodies were purchased from Cell Signaling Technology, Inc. Mouse polyclonal anti-b-actin antibody (1:1,000; catalog no. sc-47778) was purchased from Santa Cruz Biotechnology, Inc. (Dallas, TX, USA). Primary antibodies were incubated with the membranes at $4^{\circ} \mathrm{C}$ overnight. The membranes were subsequently incubated with horseradish peroxidase (HRP)-conjugated goat anti-rabbit $\operatorname{IgG}(1: 2,000$; catalog no. CW0103S; CWBiotech, Beijing, China) or goat anti-mouse $\operatorname{IgG}(1: 2,000$; catalog no. CW0102S, CWBiotech) secondary antibodies at $4^{\circ} \mathrm{C}$ for $2 \mathrm{~h}$. Bands were visualized with Enhanced Chemiluminescence reagents (CWBiotech) and the signal was detected using an ImageQuant ${ }^{\mathrm{TM}}$ LAS $4000 \mathrm{Mini}$ Biomolecular Imager (GE Healthcare Life Sciences, Chalfont, UK). Band intensities were analyzed using ImageJ software version 1.47 (National Institutes of Health, Bethesda, MD, USA) and were normalized to the band intensity of b-actin, a cell structure protein.

Statistical analysis. All results are presented as the mean \pm standard deviation of at least three experiments that were performed in triplicate. Statistical comparisons among groups were made using a one-way analysis of variance and two-tailed student's t-test. Statistical analysis was performed using SPSS 20.0 (IBM SPSS, Armonk, NY, USA). P $<0.05$ was considered to indicate a statistically significant difference.

\section{Results}

Effects of hypoxia on the expression of inflammatory cytokines and the $N F-\kappa B$ signaling pathways. The present study measured the effects of a hypoxic state on the expression levels of proinflammatory cytokines (IL-1 $\beta$, IL-6, ICAM-1, COX-2 and TNF- $\alpha$ ) in HUVECs by western blot analysis over a 24-h period. The relative expression levels of these cytokines increased significantly at the 24-h time point in hypoxic environments (Fig. 1A and B). In addition, the expression of p-p65 increased with longer exposure to hypoxia (Fig. 1A and B). These results were confirmed by ELISA on the HUVEC supernatants at $24 \mathrm{~h}$, which indicated an increased concentration of IL-6 (Fig. 1C), IL-1 $\beta$ (Fig. 1D) and TNF- $\alpha$ (Fig. 1E). These findings demonstrated that inflammatory cytokines and the NF- $\mathrm{KB}$ signaling pathway may be involved in the hypoxic response.

Silencing CLOCK inhibits hypoxia-induced inflammatory response augmentation. To confirm that hCLOCK is involved 
A

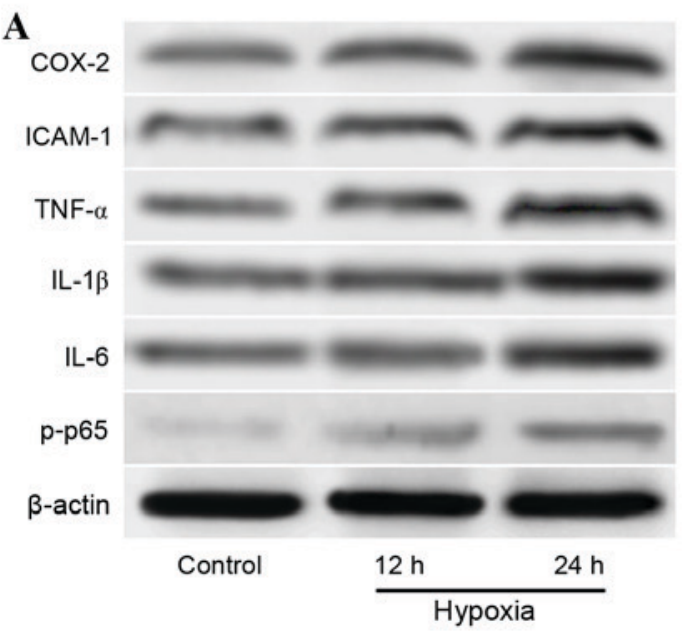

B

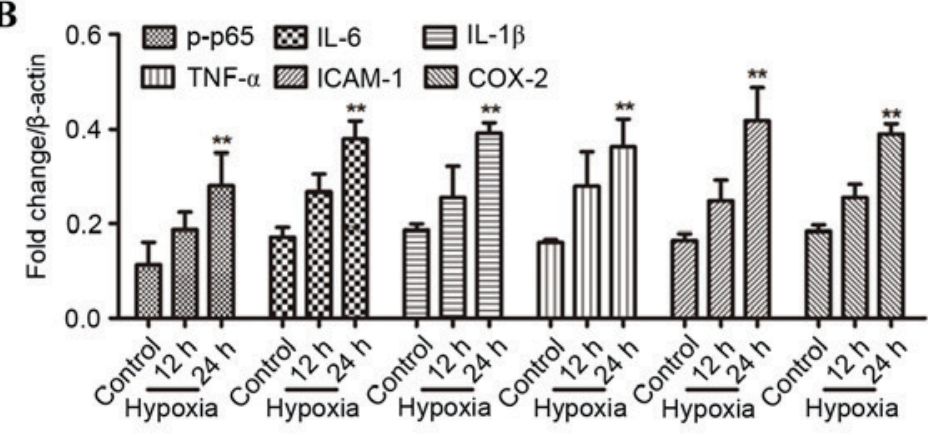

Cell supernatant

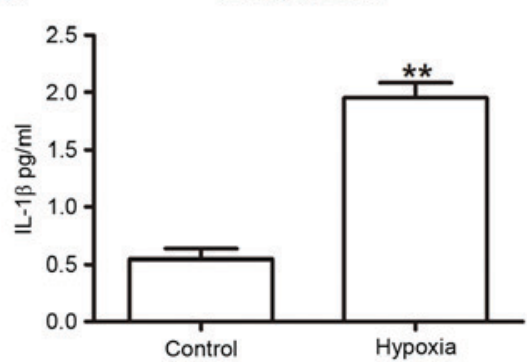

$\mathbf{E}$

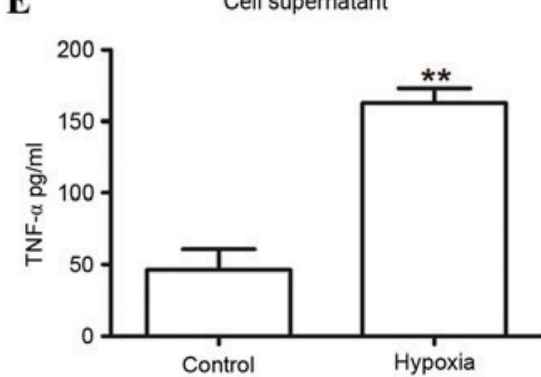

Figure 1. Hypoxia-induced expression of proinflammatory cytokines in HUVECs. (A) Western blot analysis of IL-1 $\beta$, IL-6, ICAM-1, COX-2, TNF- $\alpha$ and p-p65 expression in the HUVECs for the indicated duration. (B) Expression levels were normalized to $\beta$-actin. (C-E) ELISA analysis revealed increased supernatant concentrations of (C) IL-6, (D) IL-1 $\beta$ and (E) TNF- $\alpha$ in HUVECs with or without 24 h of hypoxia. ${ }^{* *} \mathrm{P}<0.01$ compared with control. COX-2, cyclooxygenase 2; HUVECs, human umbilical vein endothelial cells; ICAM-1, intercellular adhesion molecule 1; IL, interleukin; p-p65, phosphorylated-p65; TNF- $\alpha$, tumor necrosis factor- $\alpha$.

in hypoxia-induced inflammatory responses, hCLOCK was inhibited and the expression of inflammatory factors was evaluated. HUVECs that had been transduced with either SCR or shRNA sequences targeting hCLOCK (shCLOCK) were cultured under hypoxic conditions for $24 \mathrm{~h}$. IL-1 $\beta$, IL-6, ICAM-1, COX-2, p-p65 and TNF- $\alpha$ were significantly downregulated in the hypoxic environment when hCLOCK was knocked down compared with the SCR group $(\mathrm{P}<0.05$; Fig. 2A and B), albeit the levels were still higher than those of the control group. There was a statistically significant increase in the relative IL- 6 , TNF- $\alpha$ and IL-1 $\beta$ levels in hypoxic HUVEC supernatants compared with the normoxic control cells; however, levels were reduced in the shCLOCK group $(\mathrm{P}<0.05$; Fig. 2C-E). These findings indicated that hCLOCK is directly involved in activation of the $\mathrm{NF}-\kappa \mathrm{B}$ signaling pathway.

Effects of PDTC on hypoxia-induced inflammatory cytokine expression. The present study focused on the NF- $\mathrm{B}$ signaling pathway to determine whether $N F-\kappa B$ was a key factor in the hypoxia-mediated inflammatory response. HUVECs were subjected to hypoxia with or without exposure to the NF- $\kappa \mathrm{B}$ inhibitor PDTC. As expected, $20 \mu \mathrm{mol}$ PDTC treatment for $24 \mathrm{~h}$ significantly inhibited the expression levels of IL-1 $\beta$, IL-6, ICAM-1, COX-2, TNF- $\alpha$ and p-p65 compared with those treated with control (PBS) solution (Fig. 3A and B). Similar results were observed in ELISA analysis on the cell supernatants for IL-6 (Fig. 3C), TNF- $\alpha$ (Fig. 3D) and IL-1 $\beta$
(Fig. 3E). These findings indicated that inhibition of NF- $\kappa \mathrm{B}$ attenuates the expression of hypoxia-induced inflammatory cytokines.

$N F-\kappa B$ signaling is necessary for CLOCK-induced inflammatory cytokine expression. To further investigate whether $\mathrm{NF}-\kappa \mathrm{B}$ was crucial to the hCLOCK-mediated inflammatory response, the following culture experiments were performed: i) HUVECs were transduced with the control vector and treated with control solution (PBS); ii) HUVECs were transduced with an hCLOCK-overexpressing vector and treated with control solution; and iii) HUVECs were transduced with an hCLOCK-overexpressing vector and treated with $20 \mu \mathrm{mol}$ PDTC. The hCLOCK-overexpressing group that was treated with PBS demonstrated increased levels of IL-1 $\beta$, IL-6, ICAM-1, COX-2, p-p65 and TNF- $\alpha$ expression under hypoxic conditions (Fig. 4A-E). However, the expression levels of IL-1 $\beta$, IL-6, ICAM-1, COX-2, p-p65 and TNF- $\alpha$ expression levels were markedly decreased when hCLOCK-overexpressing HUVECs were treated with PDTC. These findings suggested that NF- $\kappa \mathrm{B}$ signaling is required for CLOCK-induced inflammatory cytokine expression.

\section{Discussion}

The present study was undertaken, in part, to elucidate the molecular basis for the hCLOCK-induced inflammatory 
A

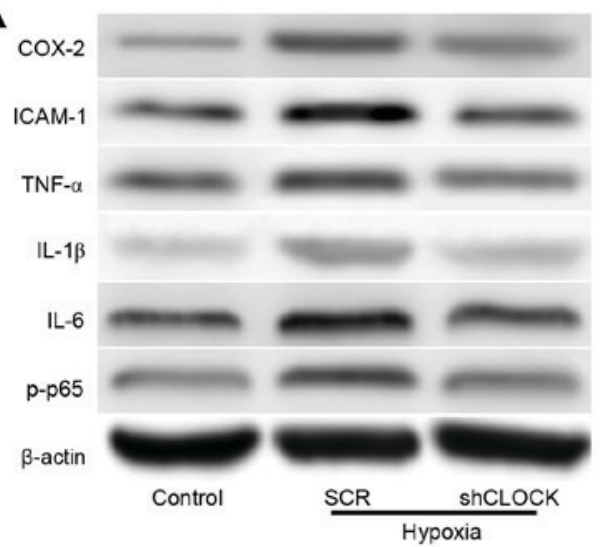

B

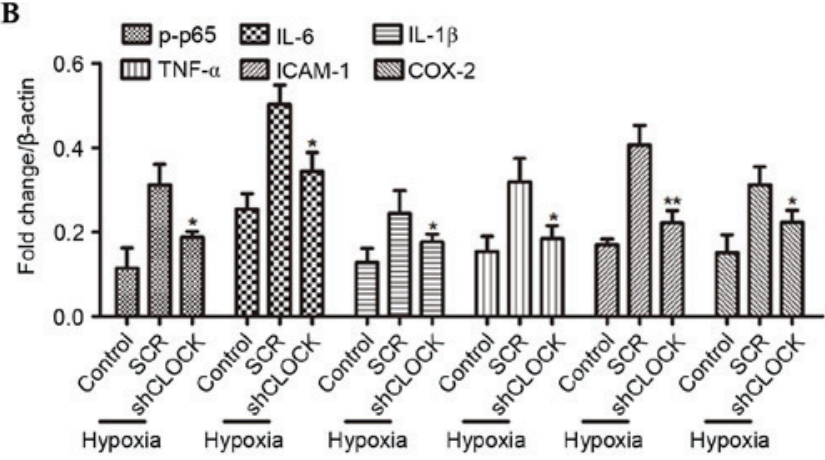

C

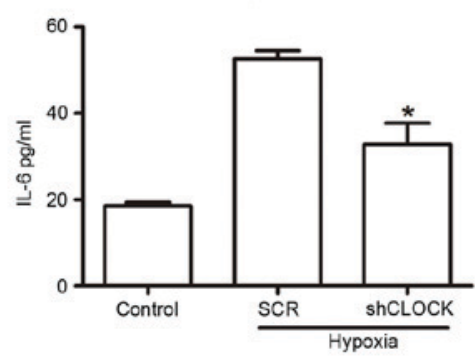

D

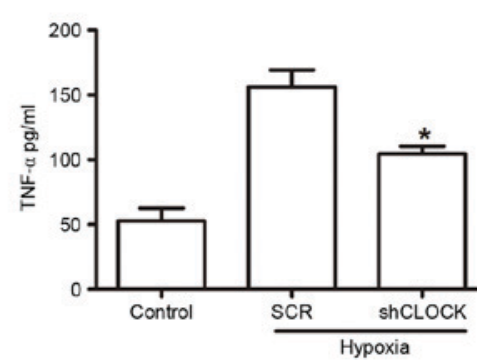

$\mathbf{E}$

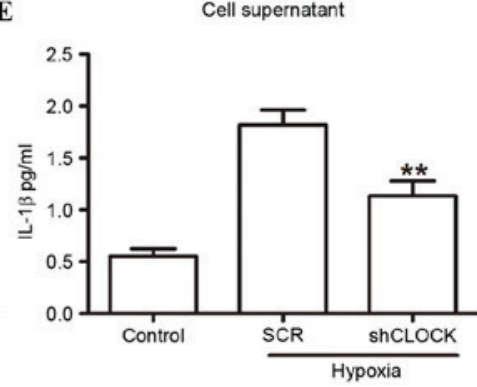

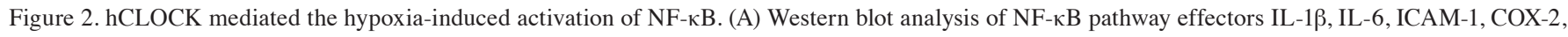
TNF- $\alpha$ and p-p65, in HUVECs that were transduced with SCR or shCLOCK vector under hypoxic conditions. (B) Expression levels are normalized to $\beta$-actin. (C-E) ELISA analysis revealed the expression levels of (C) IL-6, (D) TNF- $\alpha$ and (E) IL-1 $\beta$ in the supernatants from HUVECs that were transduced with SCR or shCLOCK vector. ${ }^{*} \mathrm{P}<0.05$ and ${ }^{* *} \mathrm{P}<0.01$ compared to SCR. COX-2, cyclooxygenase 2 ; hCLOCK, human circadian locomotor output cycle protein

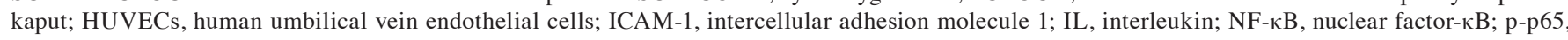
phosphorylated-p65; SCR, scrambled control vector; shCLOCK, short hairpin RNA targeting hCLOCK; TNF- $\alpha$, tumor necrosis factor- $\alpha$.

A

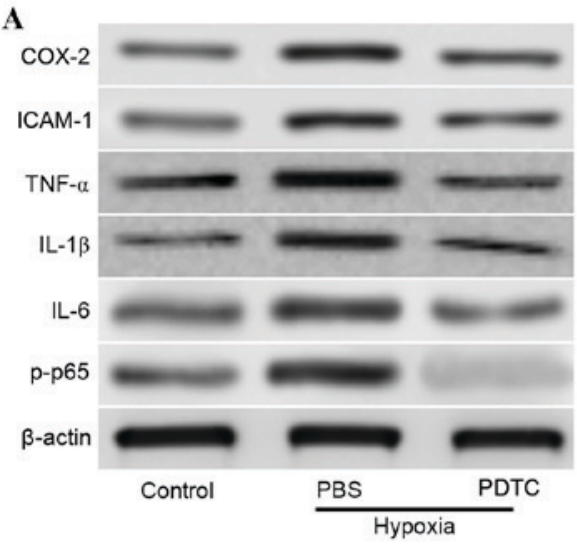

C

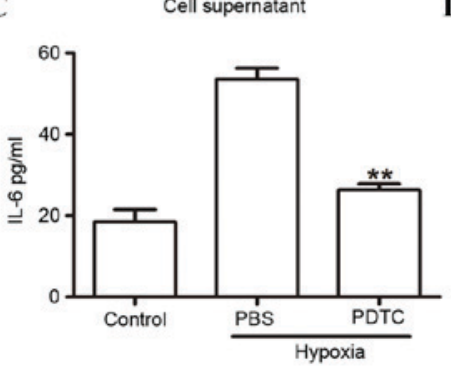

B

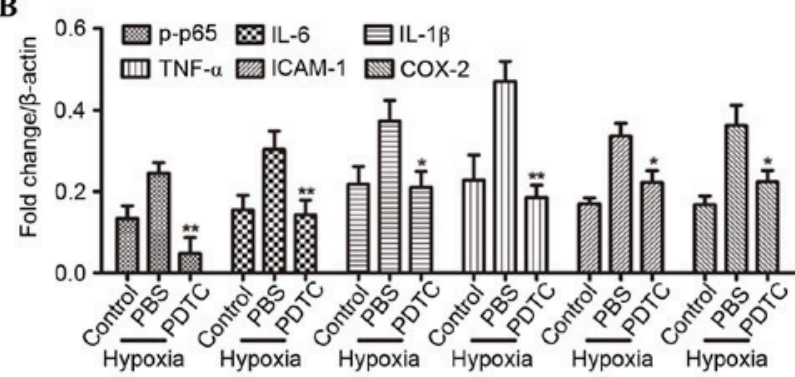

Cell supernatant

$\mathbf{E}$

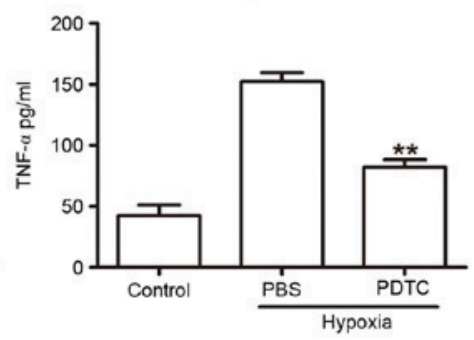

Cell supernatant

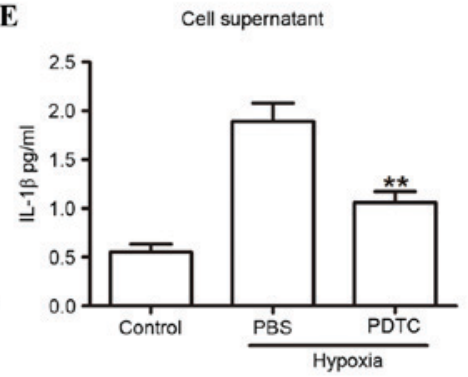

Figure 3. NF- $\mathrm{kB}$ was involved in the hypoxia-induced inflammatory response. (A) Western blot analysis of IL-1 $\beta$, IL-6, ICAM-1, COX-2, TNF- $\alpha$ and p-p65 in HUVECs that were treated with either PBS or PDTC under hypoxic conditions. (B) Expression levels are normalized to $\beta$-actin. (C-E) ELISA analysis revealed the expression levels of (C) IL-6, (D) TNF- $\alpha$ and (E) IL-1 $\beta$ in the supernatants from HUVECs that were treated with either $20 \mu$ mol PDTC or PBS under hypoxic conditions. ${ }^{*} \mathrm{P}<0.05$ and ${ }^{* *} \mathrm{P}<0.01$ compared to PBS. COX-2, cyclooxygenase 2 ; HUVECs, human umbilical vein endothelial cells; ICAM-1, intercellular adhesion molecule 1; IL, interleukin; NF-kB, nuclear factor-kB; p-p65, phosphorylated-p65; PDTC, pyrrolidine dithiocarbamate; TNF- $\alpha$, tumor necrosis factor- $\alpha$. 
A

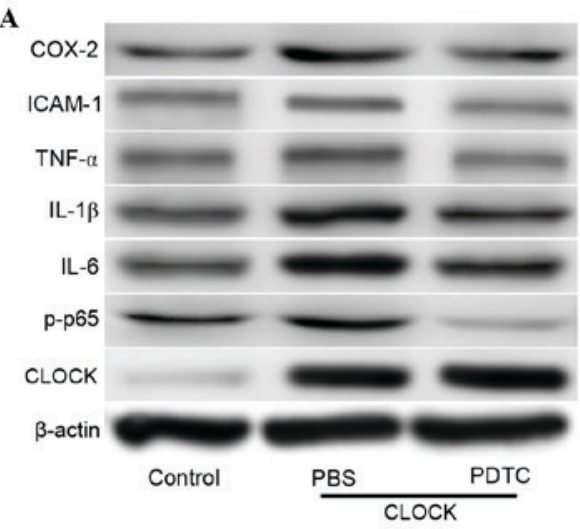

C

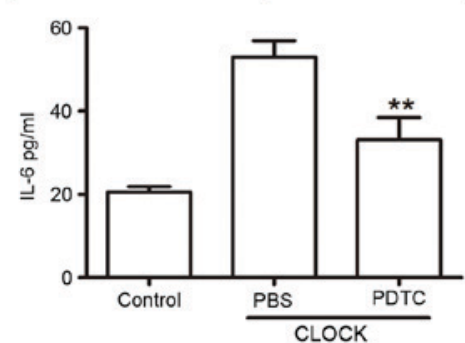

B

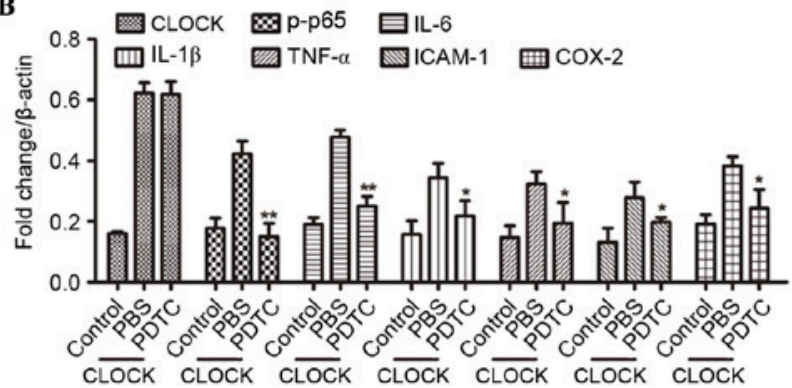

D

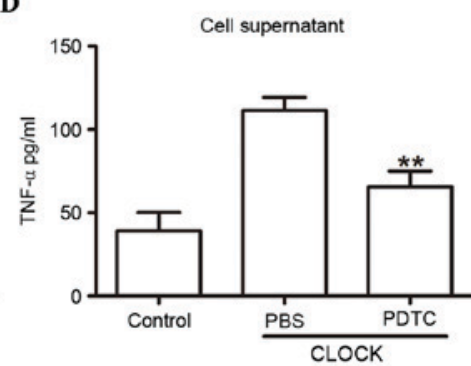

E

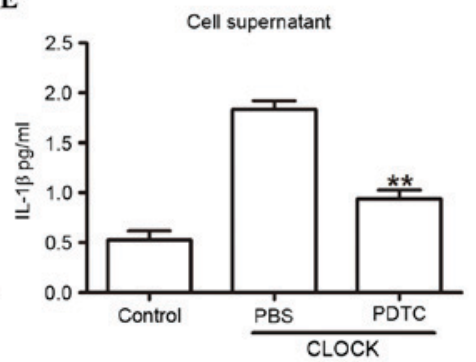

Figure 4. NF- $\kappa$ B was involved in the hCLOCK-induced inflammatory response. (A) Western blot analysis of CLOCK, IL-1 $\beta$, IL-6, ICAM-1, COX-2, TNF- $\alpha$ and p-p65 in HUVECs that were transduced with the control or hCLOCK vector, and were then treated with either PBS or $20 \mu$ mol PDTC. (B) Expression levels were normalized to $\beta$-actin. (C-E) ELISA analysis revealed the expression levels of (C) IL-6, (D) TNF- $\alpha$ and (E) IL-1 $\beta$ in HUVECs transduced with control vector or hCLOCK and then treated with either PBS or PDTC. ${ }^{*} \mathrm{P}<0.05$ and ${ }^{* *} \mathrm{P}<0.01$ compared to PBS. COX-2, cyclooxygenase 2 ; hCLOCK, human circadian locomotor output cycle protein kaput; HUVECs, human umbilical vein endothelial cells; ICAM-1, intercellular adhesion molecule 1; IL, interleukin; $\mathrm{NF}-\kappa \mathrm{B}$, nuclear factor- $\kappa \mathrm{B}$; p-p65, phosphorylated-p65; PDTC, pyrrolidine dithiocarbamate; TNF- $\alpha$, tumor necrosis factor- $\alpha$.

response pathway. Specifically, the results demonstrated that $\mathrm{NF}-\kappa \mathrm{B}$ and proinflammatory cytokine levels were increased in response to hypoxia; however, silencing hCLOCK reversed the effects and inhibited the hypoxia-induced inflammatory. In addition, suppressing NF- $\kappa \mathrm{B}$ with PDTC inhibited the proinflammatory cytokine levels in HUVECs under hypoxic conditions, and that $\mathrm{NF}-\kappa \mathrm{B}$ was required for the hCLOCK-mediated inflammatory response.

The mammalian circadian clock controls the rhythmic expression of numerous downstream genes, which are known as clock-controlled genes. Clock-controlled genes encode various proteins and profoundly influence cellular functions, including the daily rhythm for the synthesis and release of cytokines, chemokines and cytolytic factors $(15,16)$. In vivo and in vitro studies have clearly demonstrated that the circadian clock genes modulate inflammatory responses $(17,18)$; however, to date, the effects of the core circadian protein, CLOCK, on inflammation are rarely mentioned. In the present study, the silencing of hCLOCK inhibited hypoxia-induced inflammatory response augmentation and significantly downregulated all of the key effector proteins examined in a hypoxic environment. These findings indicated that hCLOCK is directly involved in the inflammatory response induced by hypoxia. The present study investigated this pathway based on several areas of prior research that have linked hCLOCK with the hypoxic and inflammatory responses in the cardiovascular system. The NF- $\kappa \mathrm{B}$ pathway has been implicated in the inflammatory response (12) and, therefore, the present study sought to identify the association between the circadian clock and the NF- $\mathrm{BB}$ pathway.
$\mathrm{NF}-\kappa \mathrm{B}$ belongs to a family of constitutive and inducible transcription factors. In the majority of cell types, $\mathrm{NF}-\kappa \mathrm{B}$ is mainly represented by the p65/p50 heterodimeric complex. The activated $\mathrm{NF}-\kappa \mathrm{B}$ complex enters the nucleus, where it binds to consensus sites in the promoters of specific genes, such as cytokines and various regulators of cellular survival and proliferation, and activates their expression (19). Research over the past decade has uncovered novel molecular links between the circadian clock and the NF- $\mathrm{B}$ pathway (20). A detailed mathematical model of NF- $\kappa \mathrm{B}$ indicated that the frequencies and amplitudes of the $\mathrm{NF}-\kappa \mathrm{B}$ oscillation depend on the strength and modes of coupling to the circadian clock $(21,22)$. Mutations in the cryptochrome gene, which encodes one of the core clock proteins, enhance extrinsic apoptosis by interfering with $\mathrm{NF}-\kappa \mathrm{B}-$ mediated transcriptional activation of genes that are required for anti-apoptosis in response to cytokine stimulation (13).

The present study confirmed an initial link between hCLOCK expression and the hypoxia-induced response pathway, and that the inhibition of $\mathrm{NF}-\kappa \mathrm{B}$ attenuates the expression of hypoxia-induced inflammatory cytokines. There are multiple hypoxic response pathways; however, the role of $\mathrm{NF}-\kappa \mathrm{B}$ signaling in hCLOCK-induced inflammation has not, to the best of our knowledge, been characterized definitively. The NF- $\mathrm{KB}$ inhibitor PDTC is a dithiocarbamate of the pyrrole derivatives (23). This molecule is able to hinder the dissociation of the inhibitory protein $\mathrm{I} \kappa \mathrm{B}$ from the $\mathrm{NF}-\kappa \mathrm{B}$ complex through antioxidation, thus inhibiting NF- $\kappa \mathrm{B}$ activation (24). In addition, PDTC impedes the translocation of p65 to the nucleus and significantly reduces the expression of p65 in the nucleus (25). 
PDTC may also directly reduce the binding ability between $\mathrm{NF}-\kappa \mathrm{B}$ and DNA, obstructing the $\mathrm{NF}-\kappa \mathrm{B}$-activated signaling pathway (26). When HUVECs cultured in hypoxic conditions were exposed to PDTC, the previously overexpressed hypoxia-induced inflammatory cytokines were downregulated. Furthermore, when the hCLOCK-overexpressing HUVECs were treated with PDTC, they exhibited decreased protein levels of IL-1 $\beta$, IL- 6 and TNF- $\alpha$, which are downstream effectors. The findings of the present study further indicated that $\mathrm{NF}-\kappa \mathrm{B}$ signaling was necessary for CLOCK-induced inflammatory cytokine expression.

The NF- $\kappa$ B pathway profoundly influences human biology. Excessive or dysregulated activation of the $N F-\kappa B$ pathway may lead to the development of pathological inflammation, which may in turn cause acute and chronic diseases. Often, chronic inflammation is linked to pathologies, such as arthritis, asthma, septic shock, lung fibrosis, glomerulonephritis, atherosclerosis and premature aging (27). To date, research has been performed on multiple links in a complex chain of biochemical pathways and has ultimately connected regulators, such as hCLOCK, with inflammation and tumorigenesis. Since $\mathrm{NF}-\kappa \mathrm{B}$ is considered to be a plausible target for therapeutic activation and suppression, the present novel characterization of the relative roles of the $\mathrm{NF}-\kappa \mathrm{B}$ signaling pathway in regulating hCLOCK-induced inflammation may allow for the development of novel therapeutic tools and strategies for treating inflammatory disorders.

In conclusion, the results of the present study linked two major signaling pathways: The circadian clock, which introduces a temporal variable into numerous physiological functions, and the NF- $\mathrm{NB}$ pathway, which is a key nodal focus in the inflammatory response. These data suggested that the mechanisms of inflammation induced by CLOCK primarily involve activation of the $\mathrm{NF}-\kappa \mathrm{B}$ signaling pathways. These findings may aid further elucidation of certain aspects of the complex biology of inflammation and tumorigenesis.

\section{Acknowledgements}

The present study was supported by grants from the National Natural Science Foundation of China (grant no. 81570433), the Project of Shanghai Municipal Commission of Health and Family Planning (grant no. 20154Y0104) and the Zhongshan Hospital Youth Talent Training Program (grant no. 201514).

\section{References}

1. Xu C, Lu C, Hua L, Jin H, Yin L, Chen S and Qian R: Rhythm changes of clock genes, apoptosis-related genes and atherosclerosis-related genes in apolipoprotein E knockout mice. Can J Cardiol 25: 473-479, 2009.

2. Takeda $\mathrm{N}$ and Maemura K: The role of clock genes and circadian rhythm in the development of cardiovascular diseases. Cell Mol Life Sci 72: 3225-3234, 2015.

3. Reppert SM and Weaver DR: Coordination of circadian timing in mammals. Nature 418: 935-941, 2002.

4. Ramin C, Devore EE, Pierre-Paul J, Duffy JF, Hankinson SE and Schernhammer ES: Chronotype and breast cancer risk in a cohort of US nurses. Chronobiol Int 30: 1181-1186, 2013.
5. Savvidis C and Koutsilieris M: Circadian rhythm disruption in cancer biology. Mol Med 18: 1249-1260, 2012.

6. Anea CB, Zhang M, Stepp DW, Simkins GB, Reed G, Fulton DJ and Rudic RD: Vascular disease in mice with a dysfunctional circadian clock. Circulation 119: 1510-1517, 2009.

7. Paschos GK and FitzGerald GA: Circadian clocks and vascular function. Circ Res 106: 833-841, 2010.

8. Tang X, Guo D, Lin C, Shi Z, Qian R, Fu W, Liu J, Li X and Fan L: Upregulation of the gene expression of CLOCK is correlated with hypoxia-inducible factor $1 \alpha$ in advanced varicose lesions. Mol Med Rep 12: 6164-6170, 2015.

9. Ghosh S, May MJ and Kopp EB: NF-kappa B and Rel proteins: Evolutionarily conserved mediators of immune responses. Annu Rev Immunol 16: 225-260, 1998.

10. Pahl HL: Activators and target genes of Rel/NF-kappaB transcription factors. Oncogene 18: 6853-6866, 1999.

11. Pasparakis M, Luedde T and Schmidt-Supprian M: Dissection of the NF-kappaB signalling cascade in transgenic and knockout mice. Cell Death Differ 13: 861-872, 2006.

12. Lee JH and Sancar A: Regulation of apoptosis by the circadian clock through NF-kappaB signaling. Proc Natl Acad Sci USA 108: 12036-12041, 2011.

13. Monje FJ, Cabatic M, Divisch I, Kim EJ, Herkner KR, Binder BR and Pollak DD: Constant darkness induces IL-6-dependent depression-like behavior through the NF- $\mathrm{KB}$ signaling pathway. J Neurosci 31: 9075-9083, 2011.

14. Tang X, Guo D, Lin C, Shi Z, Qian R, Fu W, Liu J, Li X and Fan L: hCLOCK causes Rho-Kinase-Mediated endothelial dysfunction and NF- $\mathrm{kB}-$ mediated inflammatory responses. Oxid Med Cell Longev 2015: 671839, 2015.

15. Labrecque $\mathrm{N}$ and Cermakian $\mathrm{N}$ : Circadian clocks in the immune system. J Biol Rhythms 30: 277-290, 2015.

16. Keller M, Mazuch J, Abraham U, Eom GD, Herzog ED, Volk HD, Kramer A and Maier B: A circadian clock in macrophages controls inflammatory immune responses. Proc Natl Acad Sci USA 106: 21407-21412, 2009.

17. Sato S, Sakurai T, Ogasawara J, Takahashi M, Izawa T, Imaizumi K, Taniguchi N, Ohno H and Kizaki T: A circadian clock gene, Rev-erb $\alpha$, modulates the inflammatory function of macrophages through the negative regulation of $\mathrm{Ccl} 2$ expression. J Immunol 192: 407-417, 2014.

18. Lawrence $\mathrm{T}$ and Fong $\mathrm{C}$ : The resolution of inflammation: Anti-inflammatory roles for NF-kappaB. Int J Biochem Cell Biol 42: 519-523, 2010.

19. Hayden MS, West AP and Ghosh S: NF-kappaB and the immune response. Oncogene 25: 6758-6780, 2006.

20. Spengler ML, Kuropatwinski KK, Comas M, Gasparian AV, Fedtsova N, Gleiberman AS, Gitlin II, Artemicheva NM, Deluca KA, Gudkov AV and Antoch MP: Core circadian protein CLOCK is a positive regulator of NF- $\kappa \mathrm{B}$-mediated transcription. Proc Natl Acad Sci USA 109: E2457-E2465, 2012.

21. González-Miranda JM: On the effect of circadian oscillations on biochemical cell signaling by NF-кB. J Theor Biol 335: 283-294, 2013.

22. Wang $\mathrm{X}, \mathrm{Yu} \mathrm{W}$ and Zheng L: The dynamics of NF- $\mathrm{BB}$ pathway regulated by circadian clock. Math Biosci 260: 47-53, 2015.

23. Cuzzocrea S, Chatterjee PK, Mazzon E, Dugo L, Serraino I, Britti D, Mazzullo G, Caputi AP and Thiemermann C: Pyrrolidine dithiocarbamate attenuates the development of acute and chronic inflammation. Br J Pharmacol 135: 496-510, 2002.

24. Liu SF and Malik AB: NF-kappa B activation as a pathological mechanism of septic shock and inflammation. Am J Physiol Lung Cell Mol Physiol 290: L622-L645, 2006.

25. Németh ZH, Haskó G and Vizi ES: Pyrrolidine dithiocarbamate augments IL-10, inhibits TNF-alpha, MIP-1alpha, IL-12, and nitric oxide production and protects from the lethal effect of endotoxin. Shock 10: 49-53, 1998.

26. Roy A, Jana A, Yatish K, Freidt MB, Fung YK, Martinson JA and Pahan K: Reactive oxygen species up-regulate CD11b in microglia via nitric oxide: Implications for neurodegenerative diseases. Free Radic Biol Med 45: 686-699, 2008.

27. Alonso-Fernández $\mathrm{P}$ and De la Fuente M: Role of the immune system in aging and longevity. Curr Aging Sci 4: 78-100, 2011. 